\title{
A classification-based method to estimate event-related potentials from single trial EEG
}

\author{
HUANG ZhiHua $^{1,2,3 \dagger}$, LI MingHong ${ }^{4 \dagger}$, ZHOU ChangLe $^{1,2^{*}} \&$ MA YuanYe M* $^{*}$ \\ ${ }^{1}$ Cognitive Science Department, Xiamen University, Xiamen 361005, China; \\ ${ }^{2}$ Fujian Key Laboratory of the Brain-like Intelligent Systems (Xiamen University), Xiamen 361005, China; \\ ${ }^{3}$ College of Mathematics and Computer Science, Fuzhou University, Fuzhou 350108, China; \\ ${ }^{4}$ Kunming Institute of Zoology, Chinese Academy of Sciences, Kunming 650223, China
}

Received June 24, 2011; accepted December 4, 2011

\begin{abstract}
A novel method based on machine learning is developed to estimate event-related potentials from single trial electroencephalography. This paper builds a basic framework using classification and an optimization model based on this framework for estimating event-related potentials. Then the SingleTrialEM algorithm is derived by introducing a logistic regression model, which could be obtained by training before SingleTrialEM is used, to instantiate the optimization model. The simulation tests demonstrate that the proposed method is correct and solid. The advantage of this method is verified by the comparison between this method and the Woody filter in simulation tests. Also, the cognitive test results are consistent with the conclusions of cognitive science.
\end{abstract}

classification, spatial-temporal signal model, optimization, logistic regression, SingleTrialEM

Citation: Huang Z H, Li M H, Zhou C L, et al. A classification-based method to estimate event-related potentials from single trial EEG. Sci China Life Sci, 2012, 55: 57-67, doi: 10.1007/s11427-011-4263-x

Techniques commonly used to monitor brain functions include electroencephalography (EEG), magnetoencephalography (MEG), functional magnetic resonance imaging (fMRI), positron emission tomography (PET). EEG has two significant features, i.e., high temporal resolution and no damage to humans. Most researchers believe that EEG measures voltage fluctuations resulting from ionic current flows within the neurons of the brain. Specific external stimuli on the sensory system or certain parts of the brain with certain meanings will evoke changes in the EEG called event-related potentials (ERPs). ERPs contain various components, each of which has its physiological, psychological or cognitive meaning.

ERPs are very weak signals hidden in the EEG. The am-

\footnotetext{
Contributed equally to this work

*Corresponding author (email: dozero@xmu.edu.cn; yuanma0716@vip.sina.com)
}

plitudes of all components of ERPs are much smaller than those of EEG. Usually, ERPs can be exposed from the EEG by removing spontaneous potentials with superposition and average (SA). SA has been widely applied and has led to several interesting researches [1]. However, the fact that SA results of different groups show a significant difference reveals that SA is approximately correct at best [2]. There are two fundamental assumptions for SA. One is that spontaneous signals in EEG are stationary processes with zero mean so that they could be eliminated by SA $[3,4]$. The other is that ERPs evoked by the same stimulus are also the same so that SA would not change the waveform of ERPs [4-7]. These two assumptions are not strictly valid. In addition, studies on the trial-to-trial variability of ERPs are required in neuro-physiological science, cognitive neuroscience and psychology [8,9]. This aim cannot be met by SA. Estimating ERPs from single trial EEG could provide these 
studies with more detailed data.

In 1967, Woody proposed a signal model describing the change of amplitude and latency from trial to trial and designed the match filter that might estimate latency of event related activities from single trial EEG [10]. Woody's thinking was expanded in subsequent works to consider the change of amplitudes of ERPs components so that the amplitude and latency of each trial could be estimated as parameters in a maximum likelihood framework [3,4,8,9,11]. A two-stage Bayesian method to estimate single trial ERPs was also proposed [12]. In the first stage, each of the $\mathrm{N}$ raw sweeps is processed by a filter based on 2 nd order a priori statistical information to obtain mean ERPs. In the second stage, a single-sweep estimation is dealt with by using the average ERPs estimated in the previous stage as an priori expected response.

Some methods based on analysis in the frequency domain were developed to estimate ERPs from single trial EEG [7,13,14]. Other papers [15-18] introduced wavelet analysis to deal with this problem. Independent component analysis (ICA) was also applied to estimate ERPs from single trial EEG [19,20]. This kind of method assumes EEG sources to be independent. The authors of the paper [21] estimated ERPs from single trial EEG by a combination of ICA, wavelet filtering, and multiple linear regression.

Most researchers built their ERPs estimation from single trial EEG on an assumption that spontaneous signals in EEG are stationary processes with zero mean, but this assumption is approximately right at best $[14,22,23]$. In these studies, the assumption that ERPs evoked by the same stimulus would be the same, one of the two fundamental assumptions of SA, is given up. The other assumption is still applied. The present paper totally abandons the two assumptions used in SA. It views the data measured by multiple electrodes at the same time as a vector, trains a classification model discovering the rough temporal position of components of ERPs by machine learning, and estimates the amplitude and latency of a component of ERPs by minimizing an optimization objective function derived from the classification model.

\section{Materials and methods}

\subsection{Spatial-temporal signal model}

The amplitudes and latencies of a component of ERPs in different electrodes are slightly different. This kind of difference has neurophysiological science implications. Also, a large number of experimental results indicate that spontaneous signals in EEG are not stationary processes with zero mean $[14,22,23]$. The spatial-temporal signal model, which is consistent with the above fact, is built in this paper. This model is described in eq. (1).

$$
e_{j}(t)=\sum_{r=1}^{R} \delta_{r j} \cdot s_{r}\left(t-\tau_{r j}\right)+x_{j}(t), \quad j=1, \ldots, n,
$$

where

$$
s_{r}\left(t-\tau_{r j}\right)=\exp \left(-\frac{\left(t-\tau_{r j}\right)^{2}}{2 \sigma^{2}}\right)
$$

$s_{r}\left(t-\tau_{r j}\right)$ denotes the normalized waveform of component $r$ on electrode $j ; \tau_{r j}$ denotes the latency of component $r$ on electrode $j ; \delta_{r j}$ denotes the amplitude of component $r$ on electrode $j ; e_{j}(t)$ denotes the EEG on electrode $j$; and $x_{j}(t)$ denotes the spontaneous signal in EEG on electrode $j . R$ is the number of components in ERPs and $n$ is the number of electrodes. Eq. (2), which resembles the Gaussian function, depicts a waveform similar to the normalized waveform of ERPs components. A similar approach was adopted in [5]. It should be emphasized that there are no assumptions for $x_{j}(t)$ in this model.

The estimation of ERPs in this model is by determining the amplitudes and latencies of components of ERPs. As parameters of a spatial-temporal signal model, the amplitudes of a component on different electrodes are different, and so are its latencies. This model's high consistency with the actual situation enables an accurate estimation of the amplitudes and latencies of components of ERPs from single trial EEG.

In this model $e_{j}(t)$ is known; $\sigma$ is a span factor of the waveform of a component, and $\sigma$ is a constant for a specific component of a specific subject. Therefore, this estimation is actually to determine $\delta_{r j}$ and $\tau_{r j}(r=1, \ldots, R, j=1, \ldots, n)$ under the constraints of eqs. (1) and (2) when $e_{j}(t)$ is known. Each component could be processed separately because there is very little overlap between the waveforms of different components. Since there are two parameters on each electrode, $128(=64 \times 2)$ parameters are required to be determined for one component if EEG is measured by an instrument with 64 electrodes. In fact, an instrument with more electrodes is often used. Too many parameters might give rise to computational problems.

A large number of experiments show that the amplitudes and latencies of a component of ERPs are significantly different between various brain regions, but the difference is not obvious within the same brain region. According to this fact, which is consistent with the principles of neurophysiology, we simplify the spatial-temporal signal model, intending to construct a feasible algorithm. We may consider just one ERPs component when discussing the estimation of ERPs because components of ERPs can be dealt with separately. The time range of a component of ERPs is denoted by $T$. When $t \in T$, the subscript $r$ of $\delta_{r j}$ and $\tau_{r j}$ may be omitted. Furthermore, the set of electrodes in a brain region is denoted by $\Omega$. When $t \in T$ and $j \in \Omega$, all $\tau_{j}$ may be viewed as equivalent so that we can use $\tau$ to represent all $\tau_{j}$. 
Similarly, we can use $\delta$ to represent all $\delta_{j}$. Therefore, the spatial-temporal signal model can be simplified as following during the time range of a component and within a brain region.

$$
e_{j}(t)=\delta \cdot s(t-\tau)+x_{j}(t), \quad t \in T, j \in \Omega,
$$

where

$$
s(t-\tau)=\exp \left(-\frac{(t-\tau)^{2}}{2 \sigma^{2}}\right)
$$

There are only two undetermined parameters in the simplified model.

\subsection{Framework for estimation of ERPs}

Because we make no assumptions on the spontaneous signals in EEG, the methods of [4-6] are no longer valid. This paper proposes a framework based on classification for the estimation of ERPs. This framework is depicted in Figure 1, where $S$ denotes the waveform of the component of ERPs hidden in EEG, i.e., $\delta \cdot s(t-\tau)$ in the simplified model. E in Figure 1 denotes EEG, i.e., $e_{j}(t)$ in the model. This framework views EEG at one moment as a vector denoted by $E(t)$. When the brain is in the resting state, EEG contains only spontaneous signals, i.e., $e_{j}(t)=x_{j}(t)$. We mark the vectors in this state as positive examples. When subjects are exposed to an external stimulus, EEG contains ERPs. The temporal ranges of components of ERPs are fixed. We mark the vectors in such a range as negative examples.

For each subject, a training set including positive and negative examples can be built for any ERPs component. A classifier $\Phi$ could be trained on the training set by machine learning. $\Phi(E(t))$ ranging in $(0,1)$ means the probability of $E(t)$ as a positive example. $\Phi(E(t)) \geqslant 0.5$ if $E(t)$ is a positive example, $\Phi(E(t))<0.5$ if not. The estimation of ERPs could be transformed to an optimization problem by $\Phi$. It is easy to convert eq. (3) to eq. (5):

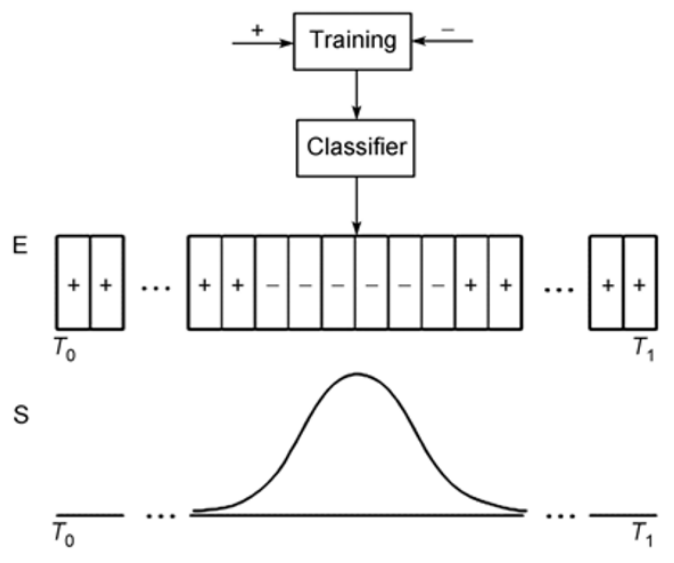

Figure 1 Framework using classification.

$$
x_{j}(t)=e_{j}(t)-\delta \cdot s(t-\tau), \quad t \in T, j \in \Omega .
$$

A vector corresponding to $x_{j}(t)$ at one moment is denoted by $X(t)$. A time range around $\tau$ is denoted by $T^{-}$. The range except $T^{-}$is denoted by $T^{+}$, i.e., $T=T^{+}+T^{-}$. When $\delta$ and $\tau$ are equal to or close to their respective real values, the following situations will happen: (i) For $t \in T^{-}$, it is not obvious for $X(t)$ to be negative examples, i.e., $\Phi(X(t))$ are very close to 0.5 ; (ii) for $t \in T^{+}, x_{j}(t)$ is almost equal to $e_{j}(t)$, i.e., $X(t)$ is almost equal to $E(t)$, since the signals of component during the time range far from the latency are very weak.

The classification result of $E(t)$ for $t \in T$ is shown as Figure 2A, where positive examples and negative examples are separated by the hyperplane $\Phi$. Eq. (5) actually expresses a kind of mapping, in which $\delta$ and $\tau$ are parameters, between the set of $X(t)$ and that of $E(t)$. The mapping between Figure 2A and Figure 2B will happen when $\delta$ and $\tau$ are equal or close to their respective real values. In Figure $2 \mathrm{~B}$, $X(t)$ corresponding to $E(t)$ as positive examples in Figure 2A have hardly been moved, but $X(t)$ corresponding to $E(t)$ as negative examples in Figure 2A have been moved close to the hyperplane $\Phi$. Based on Definition 1 (see Appendix for all Definitions and Propositions), our model is described in Eq. (6). Solving the optimization problem under the constraint of the spatial-temporal model gives the estimation of amplitude and latency of a component of ERPs from single trial EEG.

$$
\hat{\delta}, \hat{\tau}=\underset{\delta, \tau}{\arg \min }\left(\sum_{t \in T^{+}}(X(t)-E(t))^{\prime} \cdot(X(t)-E(t))+\sum_{t \in T^{-}} \Delta(X(t), \Phi)\right) .
$$

According to Definition 3 and Proposition 4, let

$$
\Delta(X(t), \Phi)=\left(\beta^{\prime} \cdot V(t)\right)^{2} .
$$

Eq. (7) is a logistic-regression-based instance of $\Delta$. Eq. (6) can be transformed to Eq. (8) by Eq. (7).

$$
\hat{\delta}, \hat{\tau}=\underset{\delta, \tau}{\arg \min }\left(\sum_{t \in T^{+}}(X(t)-E(t))^{\prime} \cdot(X(t)-E(t))+\sum_{t \in T^{-}}\left(\beta^{\prime} \cdot V(t)\right)^{2}\right) .
$$

Eq. (8) is an optimization model based on logistic regres-

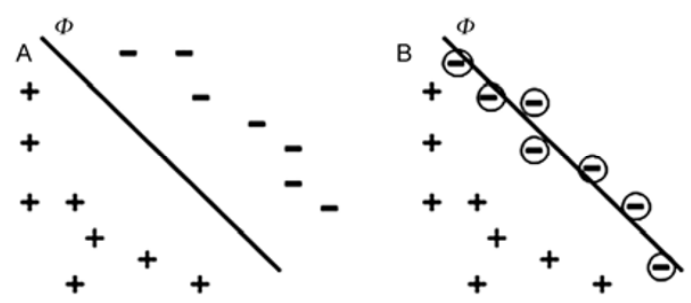

Figure 2 The method to construct the optimization objective based on the classification hyperplane. A, $E(t)$ 's distribution in the classification space. $\mathrm{B}, X(t)$ 's distribution in the classification space. 
sion, which is computable.

\subsection{SingleTrialEM algorithm}

Theoretically, any intelligent optimization algorithm can be applied to solve eq. (8). However, the general intelligent optimization algorithms do not consider the special characteristics of this model. SingleTrialEM, a special algorithm for this model, is developed in this paper. Based on Definition 4 , we have a simple form of eq. (8).

$$
\hat{\delta}, \hat{\tau}=\underset{\delta, \tau}{\arg \min } F(\delta, \tau) .
$$

According to Proposition 5, if $\tau$ takes a fixed value, then we will have

$$
F(\delta, \tau)=\lambda_{1} \delta^{2}+\lambda_{2} \delta+\lambda_{3},
$$

where $\lambda_{1}, \lambda_{2}, \lambda_{3}$ all are constants and $\lambda_{1}>0$. The solution of eq. (9) in this condition can be depicted as follows:

$$
\hat{\delta}=-\frac{\lambda_{2}}{2 \lambda_{1}}
$$

The temporal resolution of EEG and the temporal range of each ERPs component is definitive, so $\tau$ is an integer in a range when the time unit is millisecond. This, combined with Proposition 5, concludes that $F(\delta, \tau)$ is a set of quadratic polynomial functions of variable $\delta$. Therefore, $F(\delta, \tau)$ can be drawn as a group of parabolic curves as shown in Figure 3. In Figure 3, the horizontal axis represents the value of $\delta$, the vertical axis represents the value of $F(\delta, \tau)$, the three curves correspond to $F(\delta, \tau)$ of $\tau=T_{0}, T_{1}$, and $T_{2}$. Therefore, the solution of eq. (9) is in fact the minimum of a group of parabolic curves, and it is logical to propose SingleTrialEM as follows. In SingleTrialEM, $\left[T_{0}, T_{n}\right]$ is the range of $\tau$; the question of minima of Step 2 and Step 5 can be solved by eq. (11).

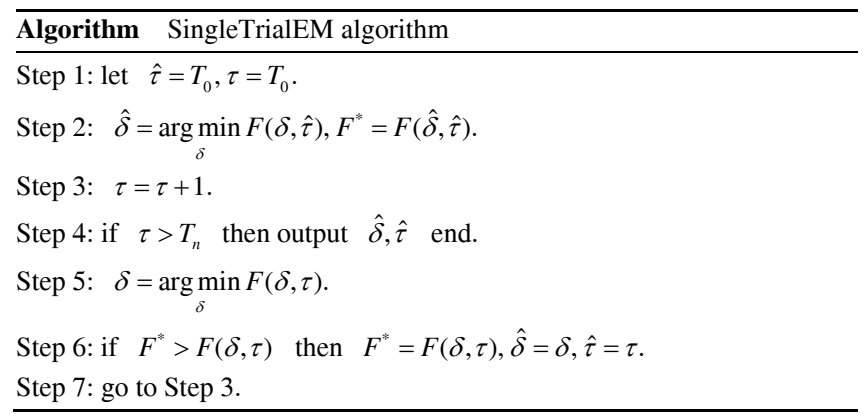

\section{Results}

\subsection{Simulation}

The procedure to build simulation data was designed by referring to [5]. The core of this procedure is the superposition of spontaneous EEG and a template of ERPs component. The real EEG recorded from subjects in the resting state could be viewed as spontaneous EEG. To collect real EEG, we randomly selected eight subjects from universities. Their age range was $19-25$, their sex ratio was $1: 1$, and all of them had normal hearing and sight. The 64 scalp electrodes were placed according to 10-20 international standards when recording EEG. The real 64-channel EEG was recorded from subjects in resting state at $1000 \mathrm{~Hz}$. The real EEG of each subject was further handled as follows.

The real EEG was divided into EEG fragments according to the standard of $200 \mathrm{~ms}$ per fragment. The EEG fragments, which were all $64 \times 200$ matrices, were viewed as $200 \mathrm{~ms}$ spontaneous signals after the stimulation. We synthesized the simulation data, which were also $64 \times 200$ matrices, by adding each channel of the EEG fragments and a template

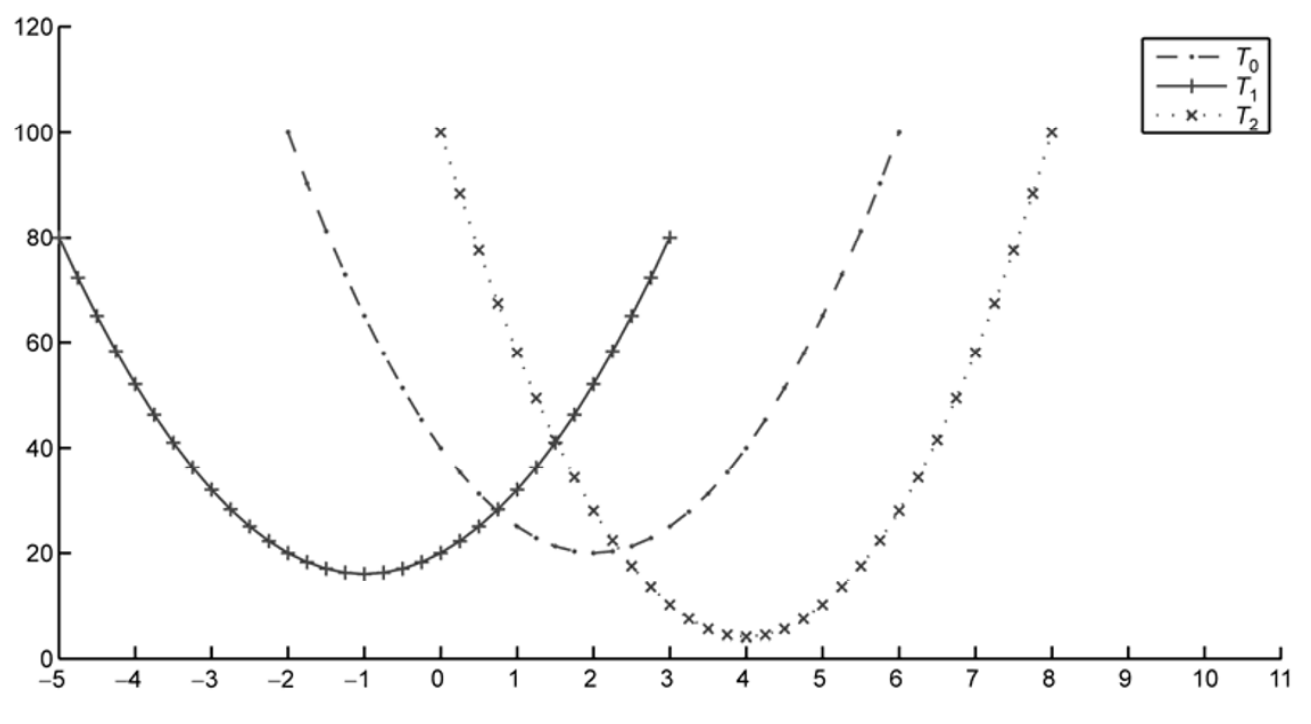

Figure 3 The $F$ function. 
of the ERPs component. Four templates of the ERPs component were generated according to eq. (4) with the span factor $\sigma$ value of $8, \tau$ value of $170 \mathrm{~ms}$ and $\delta$ respective values of $3,6,10$ and $15 \mu \mathrm{V}$. The values of $\sigma, \tau$, and $\delta$ were determined by referring to [5]. We collected 300 EEG fragments which were all synthesized respectively with four templates of the ERPs component. The simulation data were divided into four groups, each of which had the same $\delta$ value.

The vectors corresponding to a column of EEG fragments were marked as positive examples and the ones corresponding to a column of simulation data were marked as negative examples. We selected only 17 pairs of vectors corresponding to the range of 162-178 ms from one EEG fragment and its corresponding simulation data as positive and negative examples of the training set, although 200 pairs of positive and negative examples can be acquired from an EEG fragment and its corresponding simulation data. We had two reasons for doing so. First, we could get enough examples for each group of simulation data by doing so. Second, the distinction between the positive examples and negative examples near the peak of a component was obvious, while the distinction between the positive examples and negative examples far from the peak of a component was obscured. One training set was built for each of the four groups of simulation data.

For estimation of the component, we selected four brain regions, which were respectively left frontal including F1, F3, F5 and F7 channels, left parietal including P1, P3, P5 and P7 channels, right frontal including F2, F4, F6 and F8 channels, and right parietal including P2, P4, P6 and P8 channels. Each of the above four training sets was split into four according to the four brain regions. Therefore, there were 16 training sets, on which 16 models were trained. The hidden ERPs components are estimated by the SingleTrialEM algorithm with the span factor $\sigma$ value of 8 and its corresponding model.

Three examples of estimated results are presented in Figure 4. Since the ERPs component templates of the three simulation trials are same, the templates of three simulation trials could be represented by one curve (the simulation curve in Figure 4). The extracted1, extracted 2 and extracted3 curves in Figure 4 are the results estimated by SingleTrialEM respectively from three simulation trials. The results in Figure 4 are very close to their real values.

In the test, no significant difference is observed in the results of the eight subjects. We randomly select only three subjects and list their results in Tables 1,2, and 3 for brevity. The signal to noise ratio (SNR) in Tables 1,2 and 3 are calculated by

$$
S N R=20 \cdot \log \left(\frac{\sigma_{s}}{\sigma_{n}}\right),
$$

where $\sigma_{s}$ represents the standard deviation of the signal and $\sigma_{n}$ denotes the standard deviation of noise. For comparison, the results of Woody filter [10] in the four brain regions are listed in Tables 1, 2, and 3. Because the Woody filter works on one channel, we chose one channel as an alternative for a brain region, F3 for left frontal, P3 for left parietal, F4 for right frontal, and $\mathrm{P} 4$ for right parietal. The means and standard deviations of results are listed in the "Woody filter" column and the "SingleTrialEM" column of Tables 1, 2, and 3. According to the tables, SingleTrialEM does not show significantly different effects on different brain regions. The means of amplitudes and latencies estimated by SingleTrialEM are all very close to their respective real values, and their standard deviations become smaller as the SNR increases.

SingleTrialEM works much better than the Woody filter. The latencies estimated by Woody filter clearly deviate from their real values. Even in the groups with the largest SNR, differences of 4-5 ms exist between the means of latencies estimated by Woody filter and their real value, and the standard deviations are close to $60 \mathrm{~ms}$. However, SingleTrialEM could obtain satisfactory results in the groups with amplitudes of $6 \mu \mathrm{V}$ or larger. SingleTrialEM works increasingly well with increase of SNR. In the four brain

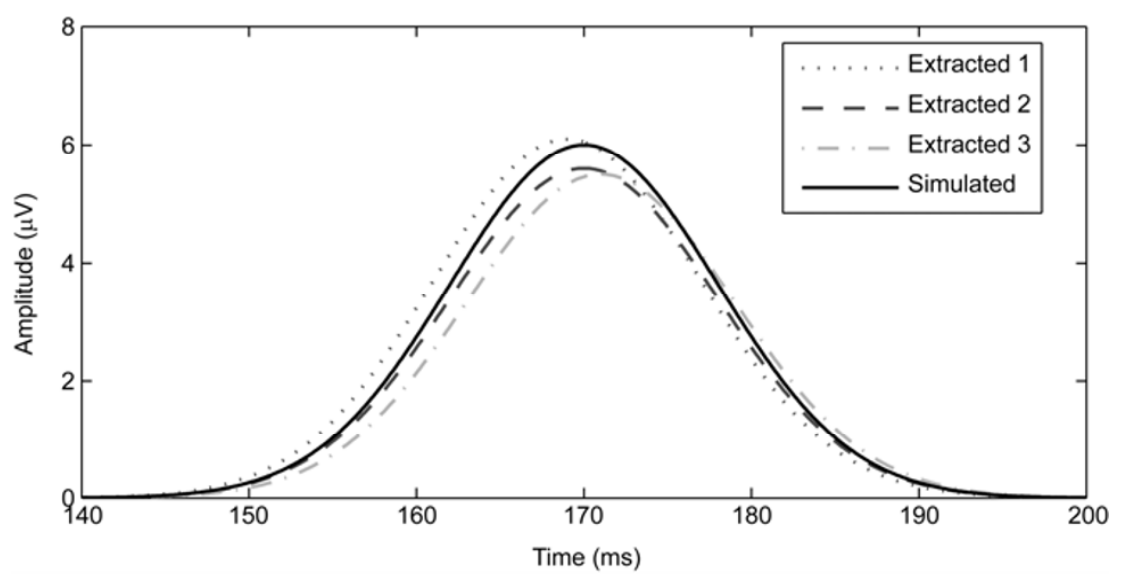

Figure 4 The examples of estimated results. 
Table 1 The results of simulation tests of subject 1

\begin{tabular}{|c|c|c|c|c|c|c|}
\hline \multirow{2}{*}{ Region } & \multirow{2}{*}{ Component } & \multirow{2}{*}{ SNR } & \multicolumn{2}{|c|}{ Woody filter } & \multicolumn{2}{|c|}{ SingleTrialEM } \\
\hline & & & Amplitude & Latency & Latency & Amplitude \\
\hline \multirow{4}{*}{ Left frontal } & 3 & -13 & $1.3 \pm 6.1$ & $160 \pm 60$ & $2.0 \pm 3.2$ & $167 \pm 5$ \\
\hline & 6 & -10 & $2.8 \pm 5.6$ & $161 \pm 60$ & $5.5 \pm 2.1$ & $171 \pm 2$ \\
\hline & 10 & -5 & $5.3 \pm 3.8$ & $165 \pm 59$ & $9.8 \pm 1.5$ & $170 \pm 1$ \\
\hline & 15 & -2 & $9.2 \pm 3.3$ & $166 \pm 57$ & $14.8 \pm 0.7$ & $170 \pm 1$ \\
\hline \multirow{3}{*}{ Right frontal } & 3 & -13 & $1.1 \pm 6.3$ & $159 \pm 62$ & $1.9 \pm 3.3$ & $172 \pm 3$ \\
\hline & 6 & -10 & $2.9 \pm 5.8$ & $161 \pm 61$ & $5.6 \pm 1.9$ & $169 \pm 2$ \\
\hline & 15 & -2 & $9.5 \pm 3.2$ & $165 \pm 57$ & $14.9 \pm 0.8$ & $170 \pm 1$ \\
\hline \multirow{4}{*}{ Left parietal } & 3 & -13 & $1.3 \pm 6.2$ & $158 \pm 60$ & $1.8 \pm 3.3$ & $167 \pm 5$ \\
\hline & 6 & -10 & $2.9 \pm 5.6$ & $160 \pm 61$ & $5.4 \pm 2.3$ & $172 \pm 3$ \\
\hline & 10 & -5 & $5.6 \pm 3.7$ & $163 \pm 59$ & $9.7 \pm 1.6$ & $170 \pm 2$ \\
\hline & 15 & -2 & $9.1 \pm 3.2$ & $165 \pm 56$ & $14.8 \pm 0.6$ & $170 \pm 1$ \\
\hline \multirow{3}{*}{ Right parietal } & 3 & -13 & $1.2 \pm 6.3$ & $161 \pm 62$ & $1.9 \pm 3.4$ & $168 \pm 5$ \\
\hline & 6 & -10 & $2.6 \pm 5.5$ & $160 \pm 60$ & $5.5 \pm 2.3$ & $169 \pm 2$ \\
\hline & 10 & -5 & $5.6 \pm 3.9$ & $165 \pm 58$ & $9.8 \pm 1.4$ & $169 \pm 1$ \\
\hline
\end{tabular}

Table 2 The results of simulation tests of subject 2

\begin{tabular}{|c|c|c|c|c|c|c|}
\hline \multirow{2}{*}{ Region } & \multirow{2}{*}{ Component } & \multirow{2}{*}{ SNR } & \multicolumn{2}{|c|}{ Woody filter } & \multicolumn{2}{|c|}{ SingleTrialEM } \\
\hline & & & Amplitude & Latency & Amplitude & Latency \\
\hline \multirow{3}{*}{ Left frontal } & 3 & -13 & $1.4 \pm 6.0$ & $161 \pm 61$ & $2.0 \pm 3.1$ & $166 \pm 5$ \\
\hline & 6 & -10 & $2.7 \pm 5.7$ & $162 \pm 60$ & $5.6 \pm 2.2$ & $172 \pm 2$ \\
\hline & 15 & -2 & $9.3 \pm 3.3$ & $166 \pm 56$ & $14.7 \pm 0.8$ & $170 \pm 2$ \\
\hline \multirow{3}{*}{ Right frontal } & 3 & -13 & $1.3 \pm 6.4$ & $159 \pm 63$ & $1.9 \pm 3.5$ & $172 \pm 4$ \\
\hline & 6 & -10 & $2.8 \pm 5.9$ & $162 \pm 62$ & $5.5 \pm 1.9$ & $169 \pm 3$ \\
\hline & 15 & -2 & $9.7 \pm 3.1$ & $165 \pm 56$ & $14.8 \pm 0.9$ & $170 \pm 1$ \\
\hline \multirow{4}{*}{ Left parietal } & 3 & -13 & $1.1 \pm 6.5$ & $157 \pm 61$ & $1.8 \pm 3.4$ & $167 \pm 6$ \\
\hline & 6 & -10 & $2.7 \pm 5.7$ & $160 \pm 60$ & $5.4 \pm 2.2$ & $172 \pm 4$ \\
\hline & 10 & -5 & $5.5 \pm 3.8$ & $162 \pm 58$ & $9.6 \pm 1.7$ & $170 \pm 3$ \\
\hline & 15 & -2 & $9.2 \pm 3.3$ & $165 \pm 57$ & $14.8 \pm 0.7$ & $170 \pm 2$ \\
\hline \multirow{3}{*}{ Right parietal } & 3 & -13 & $1.2 \pm 6.2$ & $161 \pm 63$ & $1.8 \pm 3.3$ & $167 \pm 5$ \\
\hline & 6 & -10 & $2.7 \pm 5.6$ & $162 \pm 61$ & $5.5 \pm 2.4$ & $168 \pm 3$ \\
\hline & 10 & -5 & $5.5 \pm 3.8$ & $165 \pm 59$ & $9.7 \pm 1.5$ & $169 \pm 2$ \\
\hline
\end{tabular}

Table 3 The results of simulation tests of subject 3

\begin{tabular}{|c|c|c|c|c|c|c|}
\hline \multirow{2}{*}{ Region } & \multirow{2}{*}{ Component } & \multirow{2}{*}{ SNR } & \multicolumn{2}{|c|}{ Woody filter } & \multicolumn{2}{|c|}{ SingleTrialEM } \\
\hline & & & Amplitude & Latency & Amplitude & Latency \\
\hline \multirow{4}{*}{ Left frontal } & 3 & -13 & $1.3 \pm 6.2$ & $160 \pm 61$ & $2.0 \pm 3.1$ & $166 \pm 5$ \\
\hline & 6 & -10 & $2.8 \pm 5.7$ & $162 \pm 60$ & $5.6 \pm 2.1$ & $171 \pm 3$ \\
\hline & 10 & -5 & $5.4 \pm 3.9$ & $165 \pm 58$ & $9.7 \pm 1.4$ & $170 \pm 2$ \\
\hline & 15 & -2 & $9.3 \pm 3.2$ & $166 \pm 56$ & $14.7 \pm 0.8$ & $170 \pm 1$ \\
\hline \multirow{3}{*}{ Right frontal } & 3 & -13 & $1.0 \pm 6.6$ & $159 \pm 63$ & $1.9 \pm 3.1$ & $172 \pm 4$ \\
\hline & 10 & -5 & $5.2 \pm 3.6$ & $163 \pm 59$ & $10.3 \pm 1.6$ & $170 \pm 1$ \\
\hline & 15 & -2 & $9.6 \pm 3.2$ & $165 \pm 57$ & $14.8 \pm 0.9$ & $170 \pm 1$ \\
\hline \multirow{4}{*}{ Left parietal } & 3 & -13 & $1.4 \pm 6.1$ & $158 \pm 61$ & $1.8 \pm 3.2$ & $166 \pm 5$ \\
\hline & 6 & -10 & $2.9 \pm 5.5$ & $160 \pm 60$ & $5.4 \pm 2.2$ & $172 \pm 4$ \\
\hline & 10 & -5 & $5.6 \pm 3.7$ & $163 \pm 59$ & $9.7 \pm 1.6$ & $170 \pm 2$ \\
\hline & 15 & -2 & $9.1 \pm 3.3$ & $165 \pm 55$ & $14.8 \pm 0.7$ & $170 \pm 1$ \\
\hline \multirow{3}{*}{ Right parietal } & 3 & -13 & $1.2 \pm 6.2$ & $161 \pm 61$ & $1.9 \pm 3.3$ & $168 \pm 4$ \\
\hline & 10 & -5 & $5.6 \pm 3.8$ & $165 \pm 59$ & $9.8 \pm 1.5$ & $169 \pm 1$ \\
\hline & 15 & -2 & $9.8 \pm 3.2$ & $166 \pm 58$ & $14.9 \pm 0.6$ & $170 \pm 1$ \\
\hline
\end{tabular}


regions, the means of latencies estimated by SingleTrialEM from the groups with amplitude of $15 \mu \mathrm{V}$ are all equal to their real values, and the standard deviation are very small. In terms of the estimation of amplitude, the results of SingleTrialEM in the groups with amplitude of $6 \mu \mathrm{V}$ are essentially better than those of Woody filter in the groups with amplitude of $10 \mu \mathrm{V}$. When the amplitudes of components are less than $6 \mu \mathrm{V}$, the means of amplitudes estimated by SingleTrialEM deviate from their real values and the standard deviations are also significantly larger. However, with the increase of SNR, the means of amplitudes estimated by SingleTrialEM gradually approach their real values, and the standard deviations become smaller and smaller.

\subsection{Cognitive test}

Sensory gating is a normal function of brain to inhibit the irrelevant stimuli. This function is a mechanism, particularly important for the brain to adapt to new environments, by which the brain filters redundant information and maintains the integrity of cognitive function [24]. Sensory gating protects the cognition by affecting the cognitive processes such as attention and working memory [25]. Attention performance is also positive correlated with sensory gating [24]. Therefore, we took the ordinary experiment pattern of researching sensory gating which is called the paired-click paradigm. Then we put this pattern into a cognitive sequence task called the delayed-response task with different memory load. We could estimate the ERPs of a single trial in different cognitive processes by SingleTrialEM.

Normally, the amplitudes of ERPs evoked by second sounds are smaller than those of ERPs evoked by first sounds. This phenomenon is described as pre-inhibition, which is seen as performance of sensory gating. According to the mechanism of sensory gating, we designed three cognitive tests to verify our method. In test 1 , the subjects were in resting state. In test 2 , the subjects were in the state of low load object working memory with different faces as objective stimuli. In test 3 , the subjects were also in the object working memory state with faces, but with more faces as the high memory load. All the subjects in these states were exposed to two consecutive sounds designed as paired-click paradigm. The paired-click paradigm results were compiled by Matlab with two identical sounds whose parameters were frequency $(1000 \mathrm{~Hz})$, intensity $(85 \mathrm{~dB})$, duration $(10 \mathrm{~ms})$, inter-stimuli interval $(500 \mathrm{~ms})$, and inter-trial interval (6-8 s). All these tests were repeated for 80 trials, and the second main components of ERPs N100 which were evoked by the sounds of paired-click paradigm were taken as the indicator.

Previous studies showed that sensory gating is a biological substrate [26-28] which always exists with normal brain function. Therefore, the amplitudes of N100 evoked by the first sounds should be obviously larger than the amplitudes of N100 evoked by the second sounds, no matter which one of the three states the subjects are in [26-28]. In our tests SingleTrialEM was used to estimate the component of N100 from single trial EEG. We verified SingleTrialEM by judging whether the amplitudes of N100 evoked by the first sounds were significantly larger than the ones corresponding to the second sounds.

Eight subjects, who were randomly selected from college students, participated in our tests. Their age range was 19-25, and sex ratio was $1: 1$. All of them had the normal hearing and sight. The subjects S1, S2, S3 and S4 were tested in test 1 and test 2, and the subjects S5, S6, S7, and S8 were tested in test 1 and test 3. The NeuroScan system was used to record EEG of subjects during these tests. NeuroScan software was used as a tool of the pretreatments such as removal of ocular artifacts, marking refused blocks, interception of EEG around the events, baseline adjustment, artificial rejection of artifacts, and low-pass filter. After pretreatment, many single trial EEG were obtained.

All single trial EEG of the subject S1 for the first sound in test 1 were gathered into Set 1; all single trial EEG of the subject $\mathrm{S} 1$ for the second sound in test 1 were gathered into Set 2; all single trial EEG of the subject $\mathrm{S} 1$ for the first sound in test 2 were gathered into Set 3, all single trial EEG of the subject $\mathrm{S} 1$ for the second sound in test 2 were gathered into Set 4;...; all single trial EEG of the subject S8 for the first sound in test 1 were gathered into Set 29; all single trial EEG of the subject S8 for the second sound in test 1 were gathered into Set 30; all single trial EEG of the subject $\mathrm{S} 8$ for the first sound in test 3 were gathered into Set 31; all single trial EEG of the subject S8 for the second sound in test 3 were gathered into Set 32 . Generally, such a set did not contain 80 but about 60 single trial EEG, since some single trial EEGs were discarded in the pretreatment.

Twenty single trial EEG were randomly selected from Set 1 to construct a training set. The negative examples were the vectors in the range of 162-178 ms of the selected single trial EEG. We selected the range of 162-178 ms because the latencies of N100 are most likely to be located in this range. The positive examples were the vectors in the range of 10-26 ms before the stimuli of the selected single trial EEG, because EEG in this range could be viewed as spontaneous. The training set contained the positive and negative examples from Set 1. A logistic regression model was trained on the training set. SingleTrialEM with the model was used to estimate the amplitude and latency of N100 for each single trial EEG of Set 1 . For Set 2, Set 3,.., Set 32, the amplitude and latency of N100 hidden in single trial EEG were also estimated in the same way.

Some results of the subject $\mathrm{S} 1$ in test 1 are presented in Figure 5. Figure 5A is a histogram of estimated latencies on FPZ, where blue corresponds to latencies of the first sounds and red corresponds to latencies of the second sounds. Figure $5 \mathrm{~B}$ is a comparison of estimated amplitudes on FPZ. In Figure $5 \mathrm{~B}$, the horizontal axis indicates the index number of the trial, the vertical axis indicates the estimated amplitude 

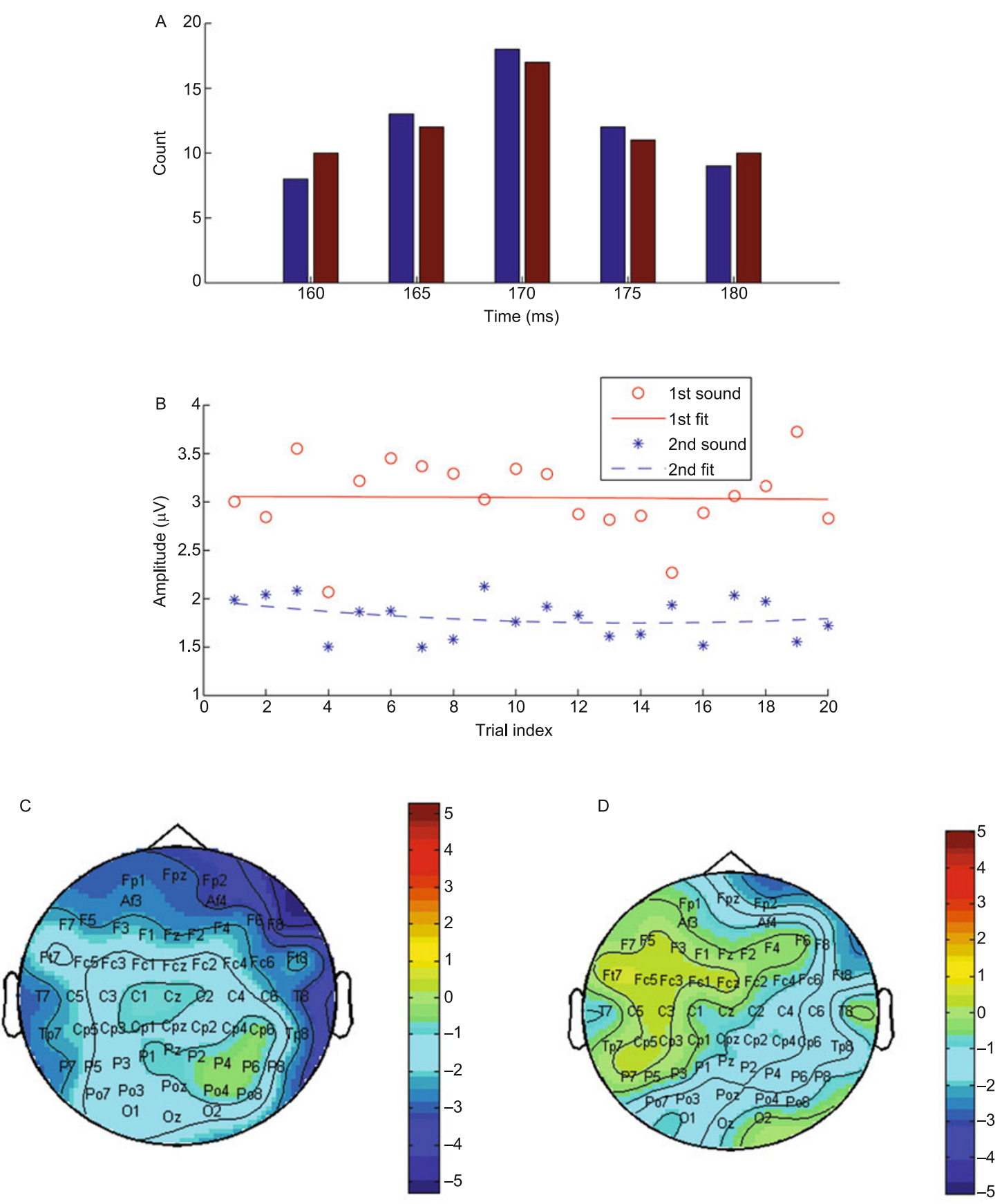

Figure 5 The results of the cognitive test. A, A histogram of estimated latencies on FPZ, where blue corresponds to latencies of the first sounds and red corresponds to latencies of the second sounds. B, A comparison of estimated amplitudes on FPZ. The horizontal axis indicates the index number of the trial and the vertical axis indicates the estimated amplitude of N100. C, The brain map drawn according to amplitudes of N100 on all channels estimated from the single trial EEG corresponding to the first sound of the tenth trial. The unit of the legend is microvolt. D, The brain map drawn according to amplitudes of

N100 on all channels estimated from the single trial EEG corresponding to the second sound of the tenth trial. The unit of the legend is microvolt.

of N100. The points represent the mean of amplitudes of three adjacent trials for the sake of conciseness, the circles indicate the amplitudes of the first sounds, and the stars denote the amplitudes of the second sounds. The solid line is a fitting of the circles, and the dotted line is a fitting of the stars. The situation in Figure 5B is consistent with sensory gating. Figure $5 \mathrm{C}$ is the brain map drawn according to amplitudes of N100 on all channels estimated from the single trial EEG corresponding to the first sound of the 10th trial. Figure 5D is the brain map drawn according to amplitudes of N100 on all channels estimated from the single trial EEG corresponding to the second sound of the 10th trial. The comparison of two brain maps is consistent with sensory gating [24,26-28]. 
Table 4 Comparison of cognitive test data

\begin{tabular}{|c|c|c|c|c|c|c|}
\hline \multirow{2}{*}{ Subject } & \multicolumn{3}{|c|}{ Test 1} & \multicolumn{3}{|c|}{ Test 2 or test 3} \\
\hline & 1st sound & 2nd sound & $P$-value & 1st sound & 2nd sound & $P$-value \\
\hline $\mathrm{S} 1$ & $3.031 \pm 2.5$ & $1.913 \pm 2.1$ & 0.015 & $2.521 \pm 2.6$ & $0.297 \pm 1.9$ & 0.009 \\
\hline $\mathrm{S} 2$ & $2.931 \pm 2.4$ & $1.767 \pm 2.2$ & 0.009 & $2.353 \pm 2.7$ & $0.325 \pm 1.7$ & 0.011 \\
\hline $\mathrm{S} 3$ & $3.281 \pm 2.6$ & $1.865 \pm 2.4$ & 0.006 & $2.581 \pm 2.5$ & $0.432 \pm 1.5$ & 0.007 \\
\hline $\mathrm{S} 4$ & $3.113 \pm 2.4$ & $1.707 \pm 2.2$ & 0.008 & $2.382 \pm 2.3$ & $0.306 \pm 1.7$ & 0.017 \\
\hline S5 & $3.291 \pm 2.7$ & $1.872 \pm 2.5$ & 0.005 & $2.573 \pm 2.4$ & $0.436 \pm 1.6$ & 0.006 \\
\hline S7 & $3.302 \pm 2.6$ & $1.873 \pm 2.4$ & 0.009 & $2.576 \pm 2.5$ & $0.425 \pm 1.5$ & 0.008 \\
\hline S8 & $3.112 \pm 2.4$ & $1.732 \pm 2.1$ & 0.006 & $2.399 \pm 2.3$ & $0.332 \pm 1.8$ & 0.015 \\
\hline
\end{tabular}

The amplitudes of N100 of each single trial EEG of these tests were estimated by SingleTrialEM. The comparison between the first sound and the second sound is listed in Table 4. The data in the "1st sound" columns are the means and standard deviations of amplitudes of N100 estimated on FPZ from all trials corresponding to the first sound. The data in the "2nd sound" columns are the means and standard deviations of amplitudes of N100 estimated on FPZ from all trials corresponding to the second sound. The amplitudes of the first sound and the second sound were matched and then tested by the signed ranks test. The data in the " $P$-value" columns are $P$-values obtained by the signed ranks test. In Table 4, the means of amplitudes of the first sound are all larger than the means of amplitudes of the second sound, and the $P$-values are all less than 0.05 . The result of Table 4 is fully consistent with sensory gating [24,26-28].

\section{Discussion and conclusion}

This paper proposes a framework based on a training classification model for estimating event-related potentials from single trial EEG. This framework is not deduced from an assumption about spontaneous EEG but from models trained on spontaneous EEG and EEG containing ERPs. This idea differs from previous ERPs estimations from single trial EEG. The SingleTrialEM algorithm was derived by introducing the logistic regression to this framework. The simulation tests show that SingleTrialEM would work excellently as long as the SNR is high enough and SingleTrialEM is superior to Woody filter. Cognitive tests based on sensory gating were designed to verify SingleTrialEM. The results of SingleTrialEM in these cognitive tests are fully consistent with sensory gating.

Introducing other classification models under this framework is worthy of further study. Additionally, several issues on our method remain to be resolved. First, the cost of training the logistic models, which must be trained before SingleTrialEM runs, is very high. Second, the span factor of SingleTrialEM is currently determined by experience. Third, it is possible that no model could be obtained by training. SingleTrialEM cannot work when no model is used. We are very interested in solving these problems in our future work.

\section{Appendix}

Definition 1. $\Delta(x, \Phi)$ is the deviation of vector $x$ to classification hyperplane $\Phi$ where $\Delta(x, \Phi)$ is a non-negative real number, and for any $x_{1}, x_{2}, \Delta\left(x_{1}, \Phi\right) \geqslant \Delta\left(x_{2}, \Phi\right)$ if $\left(\Phi\left(x_{1}\right)-0.5\right)^{2} \geqslant\left(\Phi\left(x_{2}\right)-0.5\right)^{2}$.

Definition 2. $g(z)=\frac{1}{1+\exp (-z)}, G(z)=(g(z)-0.5)^{2}$.

Definition 3. $g\left(\beta^{\prime} \cdot V(t)\right)$ is an instance of $\Phi(X(t))$, where $\beta$ is a coefficient vector of logistic regression and $V(t)=\left(1, X(t)^{\prime}\right)^{\prime}$.

Definition 4. $F(\delta, \tau)=\sum_{t \in T^{+}}(X(t)-E(t))^{\prime} \cdot(X(t)-E(t))+$ $\sum_{t \in T^{-}}\left(\beta^{\prime} \cdot V(t)\right)^{2}$

Proposition 1. $G(-z)=G(z)$.

$$
\text { Proof. } \begin{aligned}
& G(-z)=(g(-z)-0.5)^{2}=\left(\frac{1}{1+\exp (z)}-0.5\right)^{2} \\
= & \left(\frac{2}{2+2 \cdot \exp (z)}-\frac{1+\exp (z)}{2+2 \cdot \exp (z)}\right)^{2} \\
= & \left(\frac{1-\exp (z)}{2+2 \cdot \exp (z)}\right)^{2} \\
= & \left(\frac{1-\exp (-z)}{2+2 \cdot \exp (-z)}\right)^{2}=\left(\frac{2-(1+\exp (-z))}{2+2 \cdot \exp (-z)}\right)^{2} \\
= & \left(\frac{2}{2+2 \cdot \exp (-z)}-\frac{1+\exp (-z)}{2+2 \cdot \exp (-z)}\right)^{2} \\
= & \left(\frac{1}{1+\exp (-z)}-0.5\right)^{2} \\
= & (g(z)-0.5)^{2}=G(z) .
\end{aligned}
$$

Proposition 2. $G^{\prime}(z) \geqslant 0$ if $z \geqslant 0$.

$$
\text { Proof. } G^{\prime}(z)=\frac{(1-\exp (-z)) \cdot \exp (-z)}{(1+\exp (-z))^{3}},
$$

$0<\exp (-z) \leqslant 1,1-\exp (-z) \geqslant 0,(1+\exp (-z))^{3}>0$ if $z \geqslant 0$.

Therefore, $G^{\prime}(z) \geqslant 0$ if $z \geqslant 0$. 
Proposition 3. For any $z_{1}$ and $z_{2}$, if $\left(g\left(z_{1}\right)-\right.$ $0.5)^{2} \geqslant\left(g\left(z_{2}\right)-0.5\right)^{2}$ then $z_{1}^{2} \geqslant z_{2}^{2}$.

Proof. According to Definition 2, $\left(g\left(z_{1}\right)-0.5\right)^{2} \geqslant$ $\left(g\left(z_{2}\right)-0.5\right)^{2}$ is $G\left(z_{1}\right) \geqslant G\left(z_{2}\right)$.

If $z_{1} \geqslant 0$ and $z_{2} \geqslant 0$, then with $G\left(z_{1}\right) \geqslant G\left(z_{2}\right)$ from Proposition 2 we have $z_{1} \geqslant z_{2} \geqslant 0$, and further $z_{1}^{2} \geqslant z_{2}^{2}$.

If $z_{1} \geqslant 0$ and $z_{2} \leqslant 0$, then with $G\left(z_{1}\right) \geqslant G\left(z_{2}\right)$ from Proposition 1 we have $G\left(z_{1}\right) \geqslant G\left(-z_{2}\right)$. Additionally, $z_{1} \geqslant 0$ and $z_{2} \leqslant 0$ are $z_{1} \geqslant 0$ and $-z_{2} \geqslant 0$, with $G\left(z_{1}\right) \geqslant G\left(-z_{2}\right)$ from Proposition 2 we can have $z_{1} \geqslant-z_{2} \geqslant 0$ and further $z_{1}^{2} \geqslant z_{2}^{2}$.

If $z_{1} \leqslant 0$ and $z_{2} \geqslant 0$, then with $G\left(z_{1}\right) \geqslant G\left(z_{2}\right)$, from Proposition 1 we have $G\left(-z_{1}\right) \geqslant G\left(z_{2}\right)$. Additionally, $z_{1} \leqslant 0$ and $z_{2} \geqslant 0$ are $-z_{1} \geqslant 0$ and $z_{2} \geqslant 0$; with $G\left(-z_{1}\right) \geqslant G\left(z_{2}\right)$ from Proposition 2 we have $-z_{1} \geqslant z_{2} \geqslant 0$ and further $z_{1}^{2} \geqslant z_{2}^{2}$.

If $z_{1} \leqslant 0$ and $z_{2} \leqslant 0$, then with $G\left(z_{1}\right) \geqslant G\left(z_{2}\right)$ from Proposition 1 we have $G\left(-z_{1}\right) \geqslant G\left(-z_{2}\right)$. Additionally, $z_{1} \leqslant 0$ and $z_{2} \leqslant 0$ are $-z_{1} \geqslant 0$ and $-z_{2} \geqslant 0$; with $G\left(-z_{1}\right) \geqslant G\left(-z_{2}\right)$, from Proposition 2 we have $-z_{1} \geqslant-z_{2} \geqslant 0$ and further $z_{1}^{2} \geqslant z_{2}^{2}$.

To sum up, for any $z_{1}$ and $z_{2}$, if $\left(g\left(z_{1}\right)-0.5\right)^{2} \geqslant$ $\left(g\left(z_{2}\right)-0.5\right)^{2}$, then $z_{1}^{2} \geqslant z_{2}^{2}$.

Proposition 4. $\left(\beta^{\prime} \cdot V(t)\right)^{2}$ is a $\Delta$.

Proof. First, $\left(\beta^{\prime} \cdot V(t)\right)^{2}$ is a non-negative real number which can be deduced from the fact that every component of vector $\beta$ and $V(t)$ is a real number.

Second, for any $t_{1}, t_{2}, X\left(t_{1}\right), X\left(t_{2}\right)$ are equivalent to $x_{1}, x_{2}$ of Definition 1, i.e., $\left(\Phi\left(x_{1}\right)-0.5\right)^{2} \geqslant\left(\Phi\left(x_{2}\right)-0.5\right)^{2}$ can be rewritten as $\left(\Phi\left(X\left(t_{1}\right)\right)-0.5\right)^{2} \geqslant\left(\Phi\left(X\left(t_{2}\right)\right)-0.5\right)^{2}$. According to Definition 3, $\left(\Phi\left(X\left(t_{1}\right)\right)-0.5\right)^{2} \geqslant\left(\Phi\left(X\left(t_{2}\right)\right)-0.5\right)^{2}$ can be rewritten as $\left(g\left(\beta^{\prime} \cdot V\left(t_{1}\right)\right)-0.5\right)^{2} \geqslant\left(g\left(\beta^{\prime} \cdot V\left(t_{2}\right)\right)-\right.$ $0.5)^{2}$. Let $z_{1}=\beta^{\prime} \cdot V\left(t_{1}\right), z_{2}=\beta^{\prime} \cdot V\left(t_{2}\right)$, and it can be further rewritten as $\left(g\left(z_{1}\right)-0.5\right)^{2} \geqslant\left(g\left(z_{2}\right)-0.5\right)^{2}$, from which we conclude that $z_{1}^{2} \geqslant z_{2}^{2}$, i.e., $\left(\beta^{\prime} \cdot V\left(t_{1}\right)\right)^{2} \geqslant\left(\beta^{\prime} \cdot V\left(t_{2}\right)\right)^{2}$, according to Proposition 3.

Therefore, $\left(\beta^{\prime} \cdot V(t)\right)^{2}$ is a $\Delta$.

Proposition 5. If $\tau$ takes a fixed value, $F(\delta, \tau)$ is a quadratic polynomial of $\delta$ and the quadratic coefficient is greater than 0 .

Proof. According to eqs. (3) and (4), each component of vector $X(t)-E(t)$ is a monomial in $\delta$ if $\tau$ takes a fixed value. Then $(X(t)-E(t))^{\prime} \cdot(X(t)-E(t))$ is a quadratic of $\delta$ and the coefficient is greater than 0 if $\tau$ takes a fixed value.

The first component of $V(t)$ is 1 according to Definition 3. For a fixed value of $\tau$, each of other components of $V(t)$ is a polynomial in $\delta$. Each component of $\beta$ is a constant. There- fore, $\beta^{\prime} \cdot V(t)$ is a polynomial in $\delta$. Further $\left(\beta^{\prime} \cdot V(t)\right)^{2}$ is a quadratic polynomial of $\delta$ and the quadratic coefficient is greater than or equal to 0 , if $\tau$ takes a fixed value.

According to Definition 4 and the above conclusions, $F(\delta, \tau)$ is a quadratic polynomial of $\delta$ and quadratic coefficient is greater than 0 if $\tau$ takes a fixed value.

This work was supported by the National Natural Science Foundation of China (Grant No. 30670669), National Basic Research Program of China (Grant No. 2007CB947703), Natural Science Foundation of Fujian Province (Grant No. 2011J01344), and Science and Technology Development Foundation of Fuzhou University (Grant No. 2009-XQ-25).

1 Rugg M D, Coles M G H. Electrophysiology of Mind: Event-related Brain Potentials and Cognition. Oxford: Oxford University Press, 1995

2 Glaser E M, Ruchkin D S. Principles of Neurobiological Signal Analysis. New York: Academic Press, 1976

3 Jaśkowski P, Verleger R. Amplitudes and latencies of single-trial ERPs estimated by a maximum-likelihood method. IEEE Trans Biomed Eng, 1999, 46: 987-993

4 Truccolo W, Knuth K H, Shah A, et al. Estimation of single-trial multicomponent ERPs: Differentially variable component analysis (DVCA). Biol Cybern, 2003, 89: 426-438

5 Li R J, Principe J C, Bradley M, et al. A spatiotemporal filtering methodology for single-trial ERP component estimation. IEEE Trans Biomed Eng, 2009, 56: 83-92

6 Limpiti T, Veen B D V, Attias H T, et al. A spatio-temporal framework for estimating trial-to-trial amplitude variation in event-related MEG/EEG. IEEE Trans Biomed Eng, 2009, 56: 633-645

7 Melkonian D, Gordon E, Bahramali H. Single-event-related potential analysis by means of fragmentary decomposition. Biol Cybern, 2001, 85: 219-229

8 Möcks J, Gasser T, Pham D T, et al. Trial-to-trial variability of single potentials: Methodological concepts and results. Int J Neurosci, 1987, 33: 25-32

9 Truccolo W A, Ding M Z, Nakamura K H K R, et al. Trial-to-trial variability of cortical evoked responses: implications for the analysis of functional connectivity. Clin Neurophysiol, 2002, 113: 206226

10 Woody C D. Characterization of an adaptive filter for the analysis of variable latency neuroelectric signals. Med Biol Eng Comput, 1967, 5: 539-553

11 Coppolaa R, Tabor R, Buchsbaum M S. Signal to noise ratio and response variability measurements in single trial evoked potentials. Electron Clin Neuro, 1978, 44: 214-222

12 D'Avanzo C, Schiff S, Amodio P, et al. A Bayesian method to estimate single-trial event-related potentials with application to the study of the p300 variability. J Neurosci Meth, 2011, 198: 114-124

13 Tuan P D, Möcks J, Köhler W, et al. Variable latencies of noisy signals: Estimation and testing in brain potential data. Biometrika, 1987, 74: $525-533$

14 Xu L Z, Stoica P, Li J, et al. ASEO: a method for the simultaneous estimation of single-trial event-related potentials and ongoing brain activities. IEEE Trans Biomed Eng, 2009, 56: 111-121

15 Quiroga R Q, Garcia H. Single-trial event-related potentials with wavelet denoising. Clin Neurophysiol, 2003, 114: 376-390

16 Xua P, Yao D Z. Development and evaluation of the sparse decomposition method with mixed overcomplete dictionary for evoked potential estimation. Comput Biol Med, 2007, 37: 1731-1740

17 Mohseni H R, Wilding E L, Sanei S. Single trial estimation of event-related potentials using particle filtering. In: Proceedings of International Conference on Acoustics, Speech, and Signal Processing, Las Vegas, USA, 2008. 465-468

18 Mohseni H R, Nazarpour K, Wilding E L, et al. The application of 
particle filters in single trial event-related potential estimation. Physiol Meas, 2009, 30: 1101-1116

19 Lemm S, Curio G, Hlushchuk Y, et al. Enhancing the signal-to-noise ratio of ICA-based extracted ERPs. IEEE Trans Biomed Eng, 2006, 53: 601-607

20 Jung T P, Makeig S, Westerfield M, et al. Analysis and visualization of single-trial event-related potentials. Hum Brain Mapp, 2001, 14: 166-185

$21 \mathrm{Hu}$ L, Mouraux A, Hu Y, et al. A novel approach for enhancing the signal-to-noise ratio and detecting automatically event-related potentials (ERPs) in single trials. NeuroImage, 2010, 50: 99-111

22 Wang X Y, Luo C, Meng J. Nonlinear dynamical analysis of EEG and MEG: review of an emerging field. Clin Neurophysiol, 2005, 116: 2266-2301

23 Wang X Y, Luo C, Meng J. Nonlinear dynamic research on EEG signals in HAI experiment. Appl Math Comput, 2009, 207: 63-74

24 Wan L, Friedman B H, Boutros N N, et al. P50 sensory gating and attentional performance. Int J Psychophysiol, 2008, 67: 91-100

25 Lijffijt M, Lane S D, Meier S L, et al. P50, n100, and p200 sensory gating: Relationships with behavioral inhibition, attention, and working memory. Psychophysiology, 2009, 46: 1059-1068

26 Kisley M A, Noecker T L, Guinther P M. Comparison of sensory gating to mismatch negativity and self-reported perceptual phenomena in healthy adults. Psychophysiology, 2004, 41: 604-612

27 Hanlon F M, Miller G A, Thoma R J, et al. Distinct m50 and m100 auditory gating deficits in schizophrenia. Psychophysiology, 2005, 42: $417-427$

28 Oranje B, Geyer M A, Bocker K B E, et al. Prepulse inhibition and p50 suppression: Commonalities and dissociations. Psychiat Res, 2006, 143: 147-158

Open Access This article is distributed under the terms of the Creative Commons Attribution License which permits any use, distribution, and reproduction in any medium, provided the original author(s) and source are credited. 